\title{
Crystal structure of tetraaquabis(perrhenato)nickel(II), $\mathrm{Ni}\left(\mathrm{ReO}_{4}\right)_{2}\left(\mathrm{H}_{2} \mathrm{O}\right)_{4}$
}

\author{
C. Mujica \\ L'niversidad Católica del Norte. Departamento de Química. Casilla. 1280-Antofagasta. Chile \\ K. Peters, E.-M. Peters and H. G. von Schnering \\ Max-Planck-Institut für Festkörperforschung. Heisenbergstraße 1. D-70506 Stuttgar, Germany
}

Received December 20. 1996, transferred to 1st update of database ICSD in 1998. CSD-No. 402773

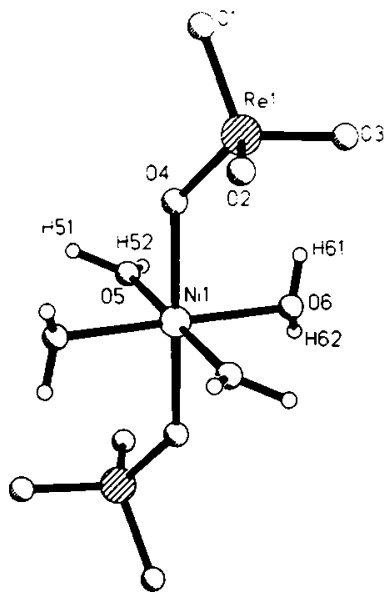

Source of material: Perrhenic acid was added dropwise to a suspension of $\mathrm{NiCO}_{3} / \mathrm{Ni}(\mathrm{OH})_{2}$ in water. The mixture was stirred and warmed up to $323 \mathrm{~K}$ until a clear green solution was formed. Green crystals of the title compound were obtained by slow evaporation of water.

The title compound crystallizes isotypically with $\mathrm{Cu}\left(\mathrm{ReO}_{4}\right)_{2}\left(\mathrm{H}_{2} \mathrm{O}\right)_{4}$ (see ref. 1). The tetrahedra around the rhenium atoms are slightly distorted. The distance of menium to the oxygen atom bound to nickel $(176(1) \mathrm{pm})$ is marginally longer than the average $\mathrm{Re}-\mathrm{O}$ distance (171(2) pm). The octahedron around nickel is almost regular with distances ranging from 203.8(9) pm to 206.1(11) pm with a mean value of 204.8(15) pm.

Table 3. Final atomic coordinates and displacement parameters (in $\AA^{2}$ )

\begin{tabular}{|c|c|c|c|c|c|c|c|c|c|c|}
\hline Atom & Site & $x$ & $y$ & $z$ & $U_{11}$ & $U_{22}$ & $U_{33}$ & $U_{12}$ & $U_{13}$ & $U_{23}$ \\
\hline $\operatorname{Re}(1)$ & $2 i$ & $0.08474(8)$ & $0.32084(8)$ & $0.29095(7)$ & $0.0241(4)$ & $0.0182(4)$ & $0.0175(3)$ & $0.0023(3)$ & $-0.0030(2)$ & $-0.0093(3)$ \\
\hline $\mathrm{Ni}(1)$ & $1 d$ & $1 / 2$ & 0 & 0 & $0.021(1)$ & $0.016(1)$ & $0.017(1)$ & $0.005(1)$ & $-0.0043(9)$ & $-0.0095(9)$ \\
\hline$O(1)$ & $2 i$ & $-0.189(2)$ & $0.325(2)$ & $0.383(2)$ & $0.029(6)$ & $0.029(6)$ & $0.029(5)$ & $0.015(5)$ & $0.002(4)$ & $-0.009(4)$ \\
\hline$O(2)$ & $2 i$ & $0.188(2)$ & $0.272(2)$ & $0.494(2)$ & $0.062(8)$ & $0.033(7)$ & $0.038(6)$ & $0.003(6)$ & $-0.020(6)$ & $-0.012(5)$ \\
\hline $\mathrm{O}(3)$ & $2 i$ & $0.125(2)$ & $0.568(2)$ & $0.103(2)$ & $0.065(8)$ & $0.040(7)$ & $0.028(5)$ & $-0.025(7)$ & $-0.009(5)$ & $-0.003(5)$ \\
\hline$O(4)$ & $2 i$ & $0.204(2)$ & $0.111(2)$ & $0.179(2)$ & $0.040(6)$ & $0.033(6)$ & $0.043(6)$ & $0.011(5)$ & $-0.018(5)$ & $-0.029(5)$ \\
\hline$O(5)$ & $2 i$ & $0.348(2)$ & $-0.075(2)$ & $-0.160(2)$ & $0.043(7)$ & $0.042(7)$ & $0.045(6)$ & $-0.008(6)$ & $-0.012(5)$ & $-0.027(5)$ \\
\hline$O(6)$ & $2 i$ & $0.514(2)$ & $0.305(2)$ & $-0.229(1)$ & $0.030(5)$ & $0.026(6)$ & $0.023(4)$ & $0.009(4)$ & $-0.004(4)$ & $-0.011(4)$ \\
\hline
\end{tabular}

Acknowledgment. C. Mujica thanks the Max-Planck-Gesellschaft for supporting a sabbatical stay at the Max-Planck-Institut für Festkörperforschung, Sturtgart.
$\mathrm{H}_{8} \mathrm{NiO}_{12} \mathrm{Re}_{2}$, triclinic, $P \overline{1}$ (No. 2), $a=6.658(1) \AA, b=6.763(1) \AA$, $c=7.161(1) \AA, \alpha=65.38(1)^{\circ}, \beta=70.27(1)^{\circ}, \gamma=71.65(1)^{\circ}, V=270.1 \AA^{3}$, $Z=1, R(F)=0.056, R_{w}(F)=0.053$.

Table 1. Parameters used for the $X$-ray data collection

$\begin{array}{ll}\text { Crystal: } & \text { pale green prism, size } 0.15 \times 0.2 \times 0.65 \mathrm{~mm} \\ \text { Wavelength: } & \text { Mo } K_{\alpha} \text { radiation }(0.71073 \AA) \\ \mu: & 241.20 \mathrm{~cm}^{-1} \\ \text { Diffractometer: } & \text { Siemens P4 } \\ \text { Scan mode: } & \omega \\ \mathrm{T}_{\text {measurement: }} & 293 \mathrm{~K} \\ 2 \theta_{\text {max: }} & 55^{\circ} \\ \mathrm{N}\left(h k h_{\text {unique: }}\right. & 1230 \\ \text { Criterion for } F_{\mathrm{o}}: & F_{\mathrm{o}}>3 \sigma\left(F_{\mathrm{o}}\right) \\ \text { N(param) } & 71 \\ \text { Program: } & \text { SHELCXTL-plus }\end{array}$

Table 2. Final atomic coordinates and displacement parameters (in $\AA^{2}$ )

\begin{tabular}{llllll}
\hline Atom & Site & $x$ & $y$ & $z$ & $U_{\text {iso }}$ \\
\hline H(51) & $2 i$ & 0.2622 & -0.1931 & -0.0588 & 0.08 \\
H(52) & $2 i$ & 0.33214 & -0.03077 & -0.26752 & 0.08 \\
H(61) & $2 i$ & 0.3828 & 0.3812 & -0.2367 & 0.08 \\
H(62) & $2 i$ & 0.5796 & 0.2952 & -0.3271 & 0.08 \\
\hline
\end{tabular}

\section{References}

1. Mujica, C.; Peters, K.; Peters, E.-M.; v. Schnering, H. G.: Crystal structure of tetraaquabis(penthenato)copper (II), $\mathrm{Cu}\left(\mathrm{ReO}_{4}\right)_{2}\left(\mathrm{H}_{2} \mathrm{O}\right)_{4}$. Z. Kristallogr. NCS 212 (1997) 294.

2. Sheldrick, G. M.: Program Package SHELXTL-plus. Release 4.1. Siemens Analytical X-Ray Instruments Inc., Madison (WI 53719), USA 1990. 